\title{
Compensación Paralelo de Reactivos Durante la Prueba de Aptitud al Cortocircuito en Transformadores de Distribución
}

\author{
Iván F. Orduña, Guillermo Aponte y Diego F. Echeverry \\ Universidad del Valle, Grupo de Investigación en Alta Tensión GRALTA, Ciudad Universitaria \\ Meléndez, Calle $13 \mathrm{~N}^{\circ}$ 100-00, Cali-Colombia. (e-mail: guillermo.aponte@correounivalle.edu.co).
}

Recibido Mar. 06, 2012; Aceptado Abr. 03, 2012; Versión final recibida Abr. 30, 2012

\section{Resumen}

En este artículo se plantea una estrategia de compensación de reactivos paralelo con capacitores, para aumentar la capacidad del campo de prueba de cortocircuito de la Universidad del Valle. Los transformadores son elementos esenciales para mantener el suministro de energía eléctrica. Por esta razón deben tener una capacidad adecuada para soportar las exigencias mecánicas, térmicas y eléctricas que se presentan durante un cortocircuito. En el laboratorio de ensayos de Alta Tensión de la Universidad del Valle (Cali. Colombia), se cuenta con un campo de prueba de cortocircuito para transformadores de distribución, el cual tiene capacidad de probar transformadores monofásicos hasta 100kVA y trifásicos hasta 300kVA. Este campo está limitado por la capacidad de corriente en la red y la prueba sólo se puede hacer a un voltaje fijo, definido por el circuito de alimentación. Los resultados muestran que la compensación paralela es una alternativa viable para reducir los requerimientos de capacidad de corriente de un campo de pruebas de cortocircuito.

Palabras clave: transformadores, cortocircuito, compensación de reactivos, capacitores, laboratorio de ensayos

\section{Reactive Parallel Compensation During a Withstand Short-circuit Test in Distribution Transformers}

\begin{abstract}
This article presents a strategy for using parallel reactive compensation with capacitors to increase the capacity of the short circuit test field at the Universidad del Valle. Transformers are key elements to maintain the electrical supply service. For that reason they must have an adequate capacity to withstand the mechanical, thermal and electrical stresses that occur during a short circuit. The High Voltage testing laboratory of the Universidad del Valle (Cali, Colombia), carries out short circuit tests for single phase distribution transformers up to 100kVA and for three phase transformers up to 300kVA. The short circuit withstand test at the laboratory is limited by the distribution network current capacity and the test can only be done at a fixed voltage, defined by the electrical network. The results show that parallel reactive compensation is a good alternative to reduce capacity requirements in short circuit tests.
\end{abstract}

Keywords: transformers, short-circuit, reactive compensation, capacitors, testing laboratory 


\section{INTRODUCCIÓN}

La prueba de cortocircuito franco o aptitud al cortocircuito se realiza con el propósito de verificar la capacidad de los transformadores de distribución para soportar las exigencias mecánicas, térmicas y eléctricas que se presentan durante un cortocircuito franco (Fogelberg, 2008). Desde el año 1994 esta prueba es requisito para la certificación de los transformadores que se instalan en las redes de distribución de las empresas de servicio eléctrico en Colombia.

La prueba requiere la circulación de corrientes muy elevadas estando el transformador energizado al voltaje nominal, lo que implica tener una fuente de potencia adecuada. Tradicionalmente, la prueba se realiza en laboratorios de alta potencia, con generadores que garantizan el suministro de una gran corriente a la tensión nominal del transformador (Fogelberg et al., 2009).

Durante el cortocircuito de un transformador de distribución, se pueden alcanzar corrientes del orden de los miles de ampers en el lado de baja tensión; sin embargo en el lado de alta tensión la corriente puede ser del orden de los cientos de ampers, estando estos valores limitados básicamente por la impedancia de cortocircuito (Zcc) de los transformadores. Durante el cortocircuito de un transformador monofásico de 13200/240V-100kVA con una Zcc de 3\%, la corriente en el lado de alta tensión es aproximadamente de 250A, mientras en el lado de baja tensión la corriente seria cercana a 13kA. Lo mencionado anteriormente, fue la base para implementar campos de prueba de cortocircuito como el de Universidad del Valle en Colombia o el de la Universidad de Sao Paulo en Brasil, los cuales emplean como fuente de cortocircuito una red de distribución en media tensión (Aponte et al., 1997; Jardinetti, 2002). Sin embargo, este tipo de fuente está limitada por la potencia que puede suministrar la red.

La respuesta en frecuencia de un transformador en cortocircuito presenta un punto de resonancia a una frecuencia mayor de $60 \mathrm{~Hz}$, donde la impedancia es muy alta y predominantemente resistiva (Aponte et al., 2011). Para que dicho estado de resonancia se sitúe a $60 \mathrm{~Hz}$, es necesario modificar el circuito equivalente visto en bornes del transformador, lo cual en la práctica se puede lograr instalando condensadores en paralelo con el transformador. Esta condición de alta impedancia y bajos reactivos, reduce la potencia suministrada por la fuente durante la prueba de cortocircuito (Maitra y Santoso, 2005). El poder aumentar el alcance de la prueba de cortocircuito en la Universidad del Valle, trae impactos importantes para Colombia y sus países vecinos, debido a que actualmente es uno de los pocos laboratorios disponibles en Suramérica para certificar la calidad de uno de los equipos más importantes de las redes eléctricas de distribución.

\section{OBTENCION DE PARAMETROS DE CORTOCIRCUITO EN TRANSFORMADORES}

\section{Circuito equivalente del transformador en régimen de cortocircuito}

El circuito equivalente serie del transformador en cortocircuito a $60 \mathrm{~Hz}$ es una rama R-L como la mostrada en la Fig.1a, del cual se puede obtener el circuito equivalente R-L paralelo observado en la Fig.1b, garantizando la misma disipación de potencia en ambos circuitos.

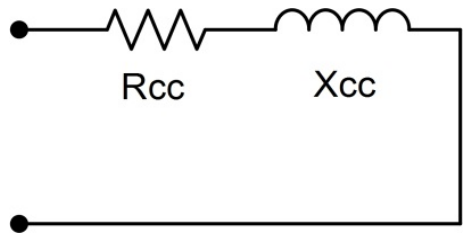

a) Equivalente serie

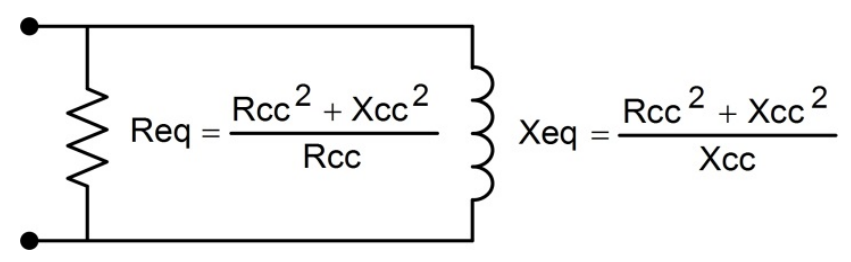

b) Equivalente paralelo

Fig.1: Equivalente circuital del transformador en cortocircuito 
Donde,

$$
\mathrm{Zcc}=\mathrm{Rcc}+\mathrm{j} \mathrm{Xcc}[\Omega]
$$

El valor de la resistencia (Rcc) y de la inductancia de cortocircuito (Xcc), se pueden encontrar de forma práctica con los resultados obtenidos en la prueba de determinación de tensión de cortocircuito y pérdidas con carga (Martínez y de León, 2011). En esta prueba, normalmente el devanado de baja tensión del transformador está cortocircuitado y un voltaje es aplicado al devanado de alta tensión, hasta hacer circular la corriente nominal en ambos devanados. Se miden la corriente nominal (Inom), las pérdidas activas (Pcc) y el voltaje de cortocircuito (Vcc), tal como se muestra en la Fig.2.

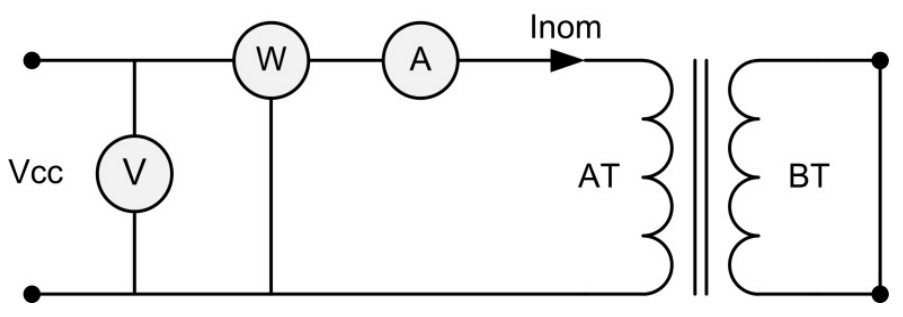

Fig.2: Diagrama de la prueba de pérdidas con carga

Con los valores medidos se calculan entonces los parámetros.

$$
\begin{aligned}
& \mathrm{Zcc}=\frac{\mathrm{Vcc}}{\operatorname{Inom}}[\Omega] \\
& \mathrm{Rcc}=\frac{\mathrm{Pcc}}{\operatorname{Inom}^{2}}[\Omega] \\
& \mathrm{Xcc}=\sqrt{\mathrm{Zcc}^{2}-\mathrm{Rcc}^{2}}[\Omega]
\end{aligned}
$$

\section{Corriente durante el cortocircuito}

Cuando un transformador es sometido a un cortocircuito franco, los primeros ciclos de la corriente son asimétricos con respecto al eje de tiempo; el grado de asimetría depende del punto en la curva de voltaje donde ocurre el cortocircuito. La corriente tiene una componente unidireccional decreciente exponencialmente a través del tiempo y una componente simétrica estable a la frecuencia fundamental del sistema (Khaparde y Kulkarni, 2004). La componente simétrica (Icc asim) depende principalmente de la impedancia de cortocircuito del transformador.

La parte asimétrica de la corriente de cortocircuito ( Icc $_{a s i m}$ ), depende de la tasa de caída de la componente exponencial, establecida por la relación Xcc/Rcc del transformador (Geno, 2011). La expresión para calcular la magnitud de esta corriente se muestra en la Ecuación 5 (NTC 532, 1999).

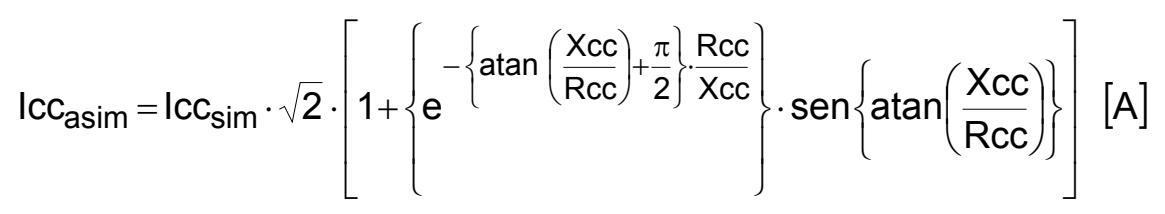

Prueba de aptitud para soportar el cortocircuito 
La comprobación de la aptitud para soportar el cortocircuito se hace comparando los valores de corriente de excitación y la impedancia de cortocircuito del transformador, antes y después de la prueba de cortocircuito (Fortin et al., 2008), estos resultados deben estar dentro de las variaciones permitidas por las normas (IEC 60076-5, 2006; IEEE C57.12.90, 2010; NTC 532, 1999). Adicionalmente, se pueden realizar algunas pruebas dieléctricas para verificar que no se haya ocasionado deterioro del aislamiento (IEC 60076-3, 2000; IEEE C57.12.90, 2010; NTC 837, 1997).

En Colombia, el procedimiento de la prueba está definido por la norma técnica colombiana NTC 532 de 1999. Se efectúan cinco pruebas de cortocircuito con una duración de $250 \mathrm{~ms}$, de estas, tres se llevan a cabo en el tap nominal, una en el de máxima tensión y una en el de mínima tensión. Una sexta prueba de cortocircuito se realiza en el tap de mínima tensión con una duración que está dada por la Ecuación 6.

$$
t=\frac{1250}{\operatorname{Icc}_{\operatorname{sim}}{ }^{2}}[s]
$$

Donde,

$\mathrm{Icc}_{\text {sim }}=$ corriente simétrica de cortocircuito $[\mathrm{pu}]$

El valor de la corriente de excitación medido después de la serie completa de pruebas de cortocircuito, no debe superar el medido antes de las pruebas en más de $25 \%$ para núcleos enrollados y $5 \%$ para apilados. La impedancia de cortocircuito medida antes de las pruebas y después de las mismas, no debe diferir en un valor superior a los porcentajes indicados en la Tabla 1 (NTC 532, 1999).

Tabla 1: Variación impedancia de cortocircuito

\begin{tabular}{|c|c|}
\hline Zcc $[\mathrm{pu}]$ & Variación permitida [\%] \\
\hline 0.0299 o menor & $22.5-(500 \times$ Zcc $)$ \\
\hline 0.0300 o mayor & 7.5 \\
\hline
\end{tabular}

\section{CÁLCULO DE LA COMPENSACION DE REACTIVOS DURANTE EL CORTOCIRCUITO}

Conectando condensadores en paralelo se puede compensar la potencia reactiva consumida por el transformador durante la prueba de cortocircuito, lo cual reduciría la corriente entregada por la fuente. En la Fig.3, se muestra el circuito paralelo del transformador con compensación.

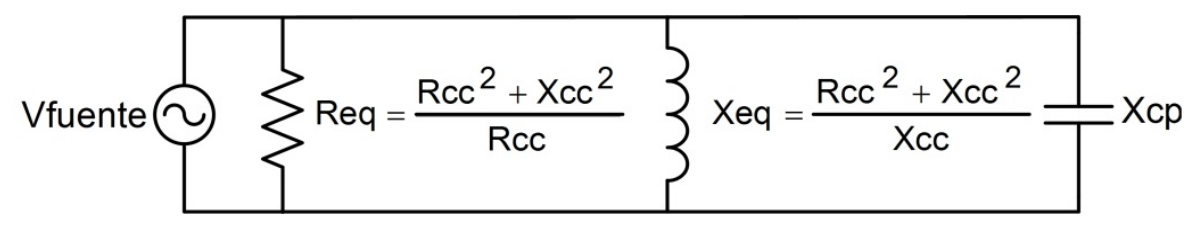

Fig.3: Compensación de reactivos paralelo

Para analizar el aporte del condensador paralelo, se encuentra la admitancia equivalente del circuito de la Fig.3, que está dada por:

$$
Y e q=G+j B=\frac{1}{R e q}+j\left(\frac{1}{X c p}-\frac{1}{X e q}\right)=\frac{R c c}{R c c^{2}+X c c^{2}}+j\left(\frac{1}{X c p}-\frac{X c c}{R c c^{2}+X c c^{2}}\right)[S]
$$


En la Ecuación 7, se observa que se puede realizar una compensación de los reactivos y llevar el circuito al estado de resonancia, conectando el valor adecuado de capacitancia (Cp), el cual está dado por la Ecuación 8.

$$
\mathrm{Cp}=\frac{\mathrm{Lcc}}{\mathrm{Rcc}^{2}+\mathrm{w}^{2} \mathrm{Lcc}^{2}}[\mathrm{~F}]
$$

La magnitud de corriente que la fuente tendría que entregar en esa condición, se calcula como:

Ifuente $=$ Vfuente $\cdot\left(\frac{\mathrm{Rcc}}{\mathrm{Rcc}^{2}+\mathrm{w}^{2} \cdot \mathrm{Lcc}^{2}}\right)[\mathrm{A}]$

Si no se logra una compensación completa, la corriente que la fuente suministra se reduce a:

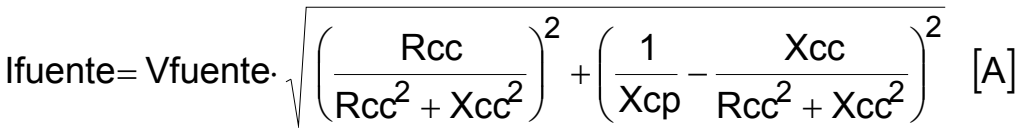

\section{VALIDACIÓN DE LA COMPENSACIÓN PARALELO EN LA PRUEBA DE CORTOCIRCUITO}

Para la validación, en este trabajo se llevó a cabo la simulación y el ensayo de cortocircuito con compensación a un transformador monofásico de 13200/244V - 15kVA a 60Hz (Fig.4).

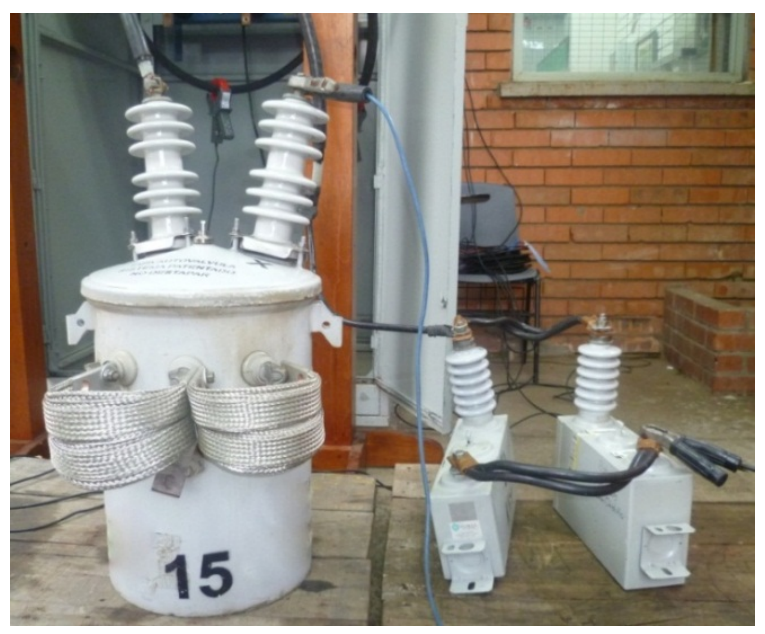

Fig.4: Transformado bajo prueba de cortocircuito

A este transformador se le realizó el ensayo de pérdidas con carga, con el fin de calcular sus parámetros de cortocircuito. Estos datos se muestran en la Tabla 2.

Tabla 2: Resultados del ensayo de pérdidas con carga y parámetros obtenidos

\begin{tabular}{|c|c|c|c|c|}
\hline \multicolumn{3}{|c|}{$\begin{array}{c}\text { Resultados del ensayo de } \\
\text { pérdidas con carga a } 25^{\circ} \mathrm{C}\end{array}$} & \multicolumn{2}{c|}{$\begin{array}{c}\text { Parámetros de } \\
\text { cortocircuito a } 25^{\circ} \mathrm{C}\end{array}$} \\
\hline $\mathrm{Pcc}$ & $\mathrm{Icc}$ & $\mathrm{Vcc}$ & $\begin{array}{c}\text { Rcc } \\
{[\Omega]}\end{array}$ & $\begin{array}{c}\text { Xcc } \\
{[\Omega]}\end{array}$ \\
\hline $\mathrm{W}]$ & {$[\mathrm{A}]$} & {$[\mathrm{V}]$} & {$[\Omega]$} & 261.09 \\
\hline 143.30 & 1.14 & 322.70 & 110.65 & 26 \\
\hline
\end{tabular}

Luego de obtener los parámetros, se calculó el valor de capacitancia necesario para la compensación de reactivos aplicando la Ecuación 8, obteniéndose una capacitancia de $8.61 \mu \mathrm{F}$. En los ensayos se utilizaron dos condensadores de 7960V a 100kVAR que se tenían en stock en el laboratorio de ensayos de Alta Tensión de la Universidad del Valle, estos elementos conectados 
en paralelo proporcionan una capacitancia equivalente de $8.37 \mu \mathrm{F}$, la cual es muy cercana a la necesaria para lograr el estado de resonancia. En la Tabla 3, se muestran las corrientes calculadas para estos valores de capacitancia, energizando el transformador a $7620 \mathrm{~V}$.

Tabla 3: Corrientes simétricas estimadas

\begin{tabular}{|c|c|c|c|}
\cline { 2 - 4 } \multicolumn{1}{c|}{} & Sin compensación & Con $8.61 \mu \mathrm{F}$ & Con $8.37 \mu \mathrm{F}$ \\
\hline Fuente $[\mathrm{A}]$ & 37.00 & 14.84 & 14.86 \\
\hline Transformador $[\mathrm{A}]$ & 37.00 & 37.00 & 37.00 \\
\hline Banco $[\mathrm{A}]$ & - & 34.97 & 34.00 \\
\hline
\end{tabular}

Según se observa en la Tabla 3 , con una capacitancia equivalente de $8.37 \mu \mathrm{F}$, se espera una disminución de más del $50 \%$ en la corriente suministrada por la fuente durante el cortocircuito.

Resultados de la simulación

Para la validación de los resultados teóricos, se empleó la herramienta SimPowerSystems del programa de cómputo MATLAB versión R2011 con el circuito mostrado en la Fig.5.

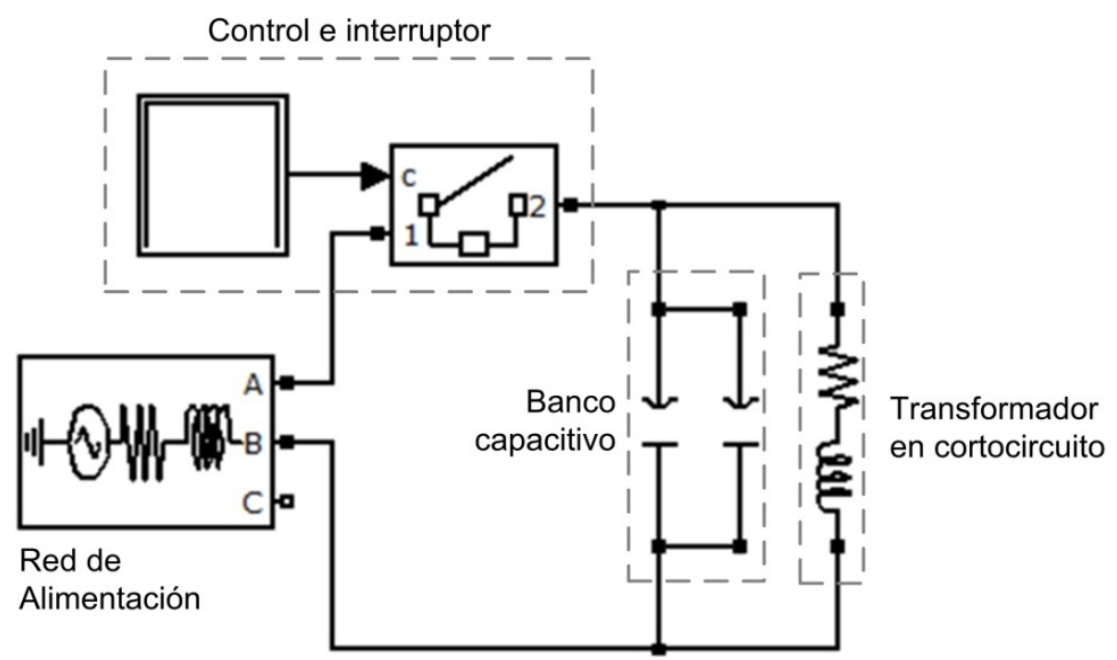

Fig.5: Circuito de simulación

Inicialmente se simuló una prueba de cortocircuito a 250ms sin compensación, obteniéndose una corriente simétrica pico de $37 \mathrm{~A}$ en el transformador, que es igual a la entregada por la fuente (Fig.6).

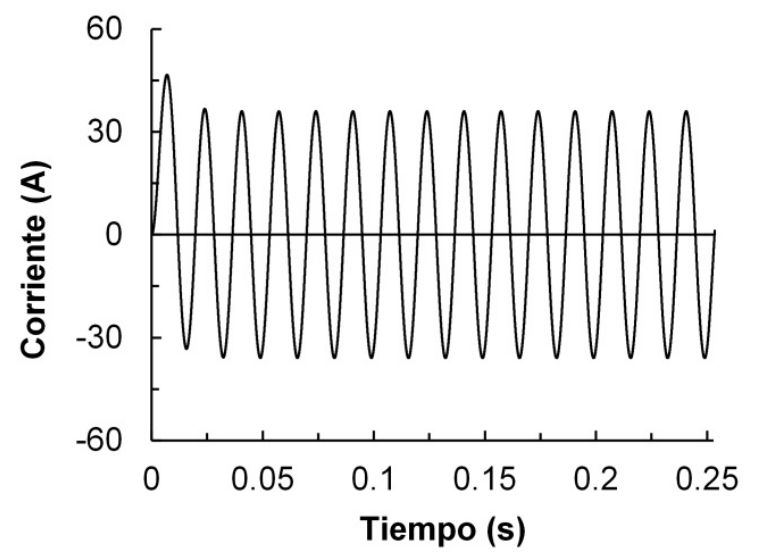

Fig.6: Corriente en la fuente sin compensación 
Posteriormente, se simuló la prueba con compensación de reactivos y se obtuvo una corriente simétrica en el transformador de 37A, mientras que la corriente en la fuente fue aproximadamente de 15A, estas corrientes son mostradas en la Fig.7.

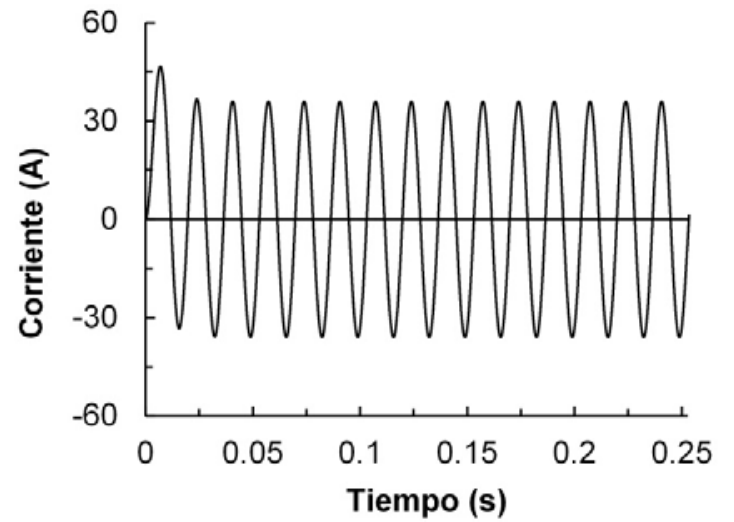

a) Transformador

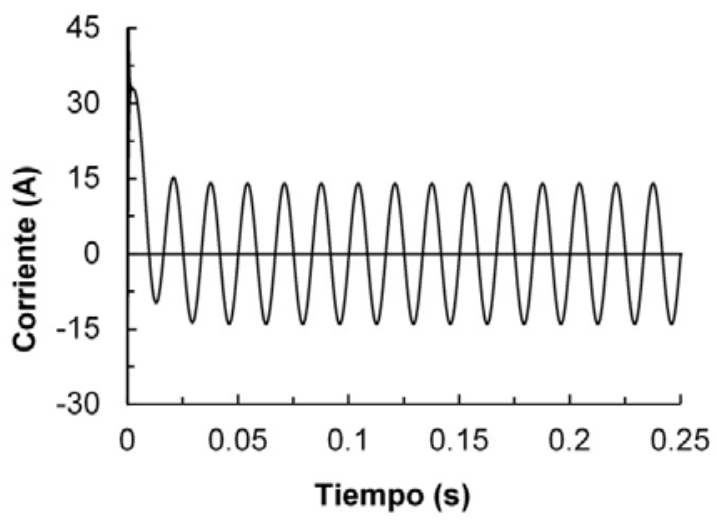

b) Fuente

Fig.7: Corrientes con compensación

En el banco capacitivo se obtuvo una corriente cercana a 33A (Fig.8).

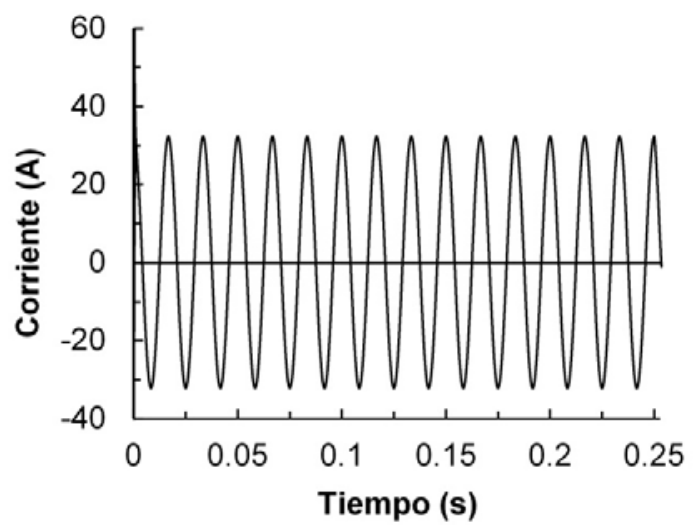

Fig.8: Corriente en el banco capacitivo durante la simulación

Resultados de la prueba

La validación práctica consistió en realizar la prueba sobre el transformador monofásico de 15kVA, inicialmente sin compensación y luego añadiendo los dos condensadores de 100kVAR en paralelo (Fig.4), con una tensión de $7620 \mathrm{~V}$ y una duración de $250 \mathrm{~ms}$. La corriente simétrica sin compensación fue de 37A (Fig.9); con compensación la corriente a través del transformador fue aproximadamente de 37A y en la fuente de 15A (Fig.10).

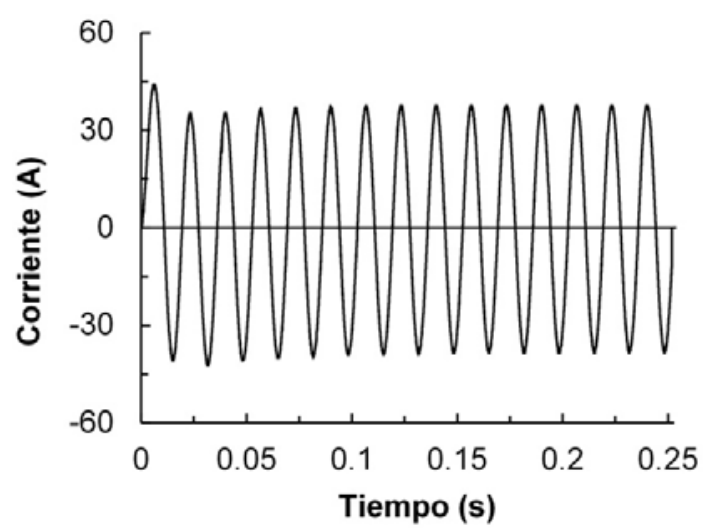

Fig.9: Corriente en la fuente sin compensación 


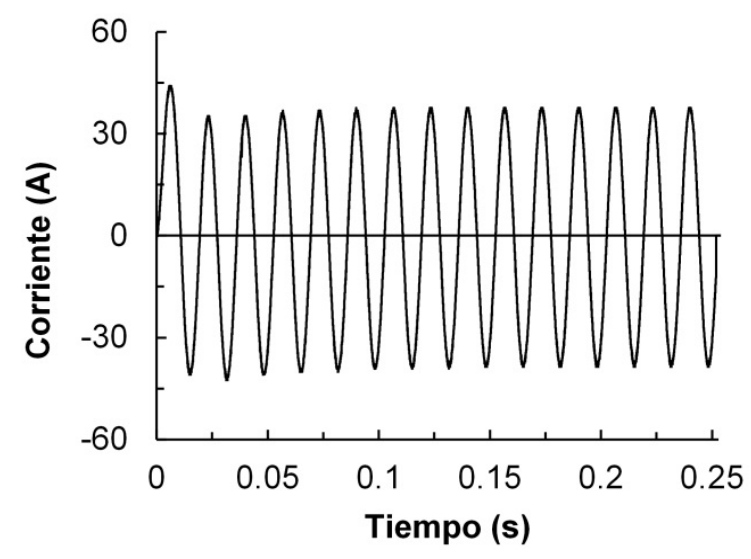

a) Transformador

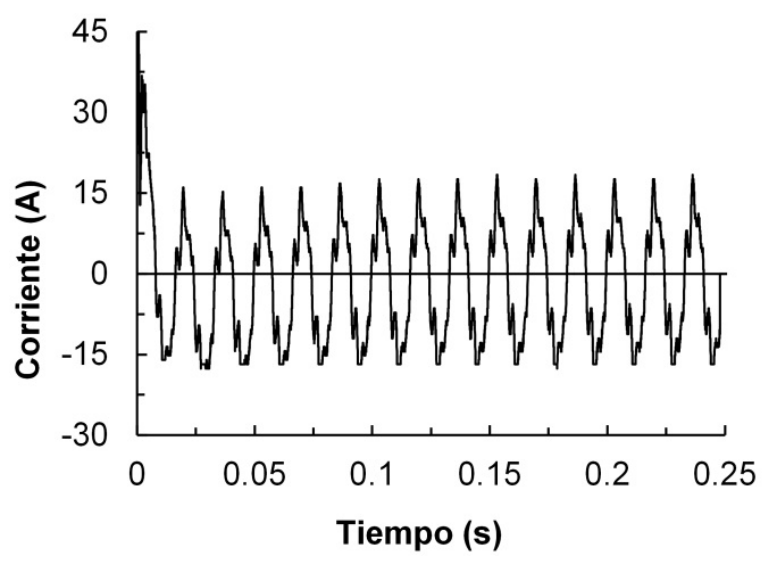

b) Fuente

Fig.10: Corrientes con compensación

La corriente registrada en el banco capacitivo fue aproximadamente 35A (Fig.11). $7.89 \times 5.5$

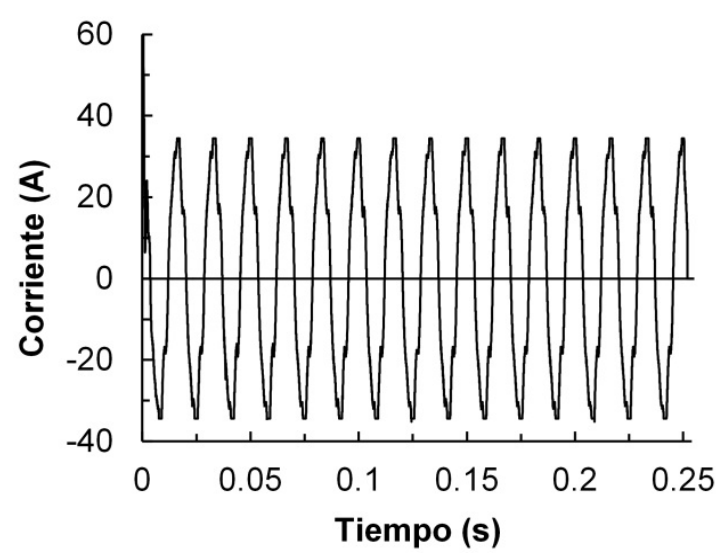

Fig.11: Corriente en el banco capacitivo durante la prueba

Las corrientes de la fuente, del transformador y del banco capacitivo durante la prueba, fueron similares a las obtenidas en la simulación. Es de resaltar que la corriente en la fuente y en el banco sufrió deformación debido a la magnificación de los armónicos presentes en la red de alimentación (Huang y Xu, 2003; Natarajan, 2005). En la Fig.12, se muestra que la distorsión armónica (THD) en la corriente suministrada por la red sin compensación fue de $0.79 \%$, mientras que con compensación de reactivos la distorsión alcanzó el $28.56 \%$.

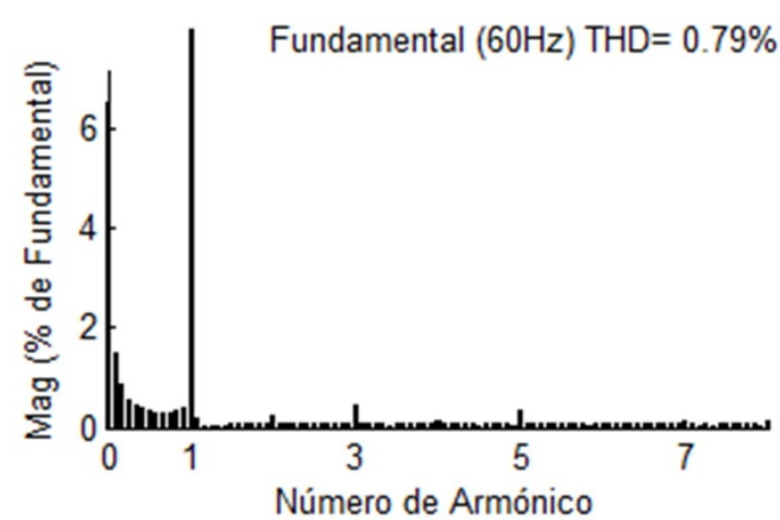

a) Sin compensación

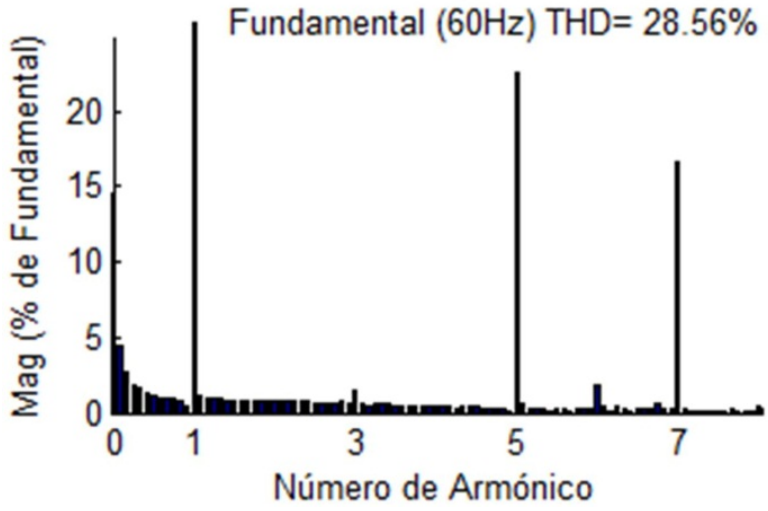

b) Con compensación

Fig.12: Distorsión armónica 
La deformación presente en la corriente, se mitiga entre la fuente y el banco capacitivo, sin afectar la corriente del transformador, tal como se observa en la Fig.10a.

\section{RESULTADOS}

En la Tabla 4, se presentan los valores de las corrientes simétricas obtenidas durante el cortocircuito en simulación y experimentalmente, con y sin compensación de reactivos.

Tabla 4: Valores de corrientes simétricas

\begin{tabular}{|c|c|c|c|c|}
\cline { 3 - 5 } \multicolumn{2}{c|}{} & $\begin{array}{c}\text { Transformador } \\
{[\mathrm{A}]}\end{array}$ & $\begin{array}{c}\text { Fuente } \\
{[\mathrm{A}]}\end{array}$ & $\begin{array}{c}\text { Banco } \\
\text { capacitivo } \\
{[\mathrm{A}]}\end{array}$ \\
\hline \multirow{2}{*}{$\begin{array}{c}\text { Sin } \\
\text { compensación }\end{array}$} & Simulación & 37.00 & 37.00 & - \\
\cline { 2 - 5 } & Prueba & 36.80 & 36.80 & - \\
\hline \multirow{2}{*}{$\begin{array}{c}\text { Con } \\
\text { compensación }\end{array}$} & Simulación & 37.00 & 15.00 & 33.00 \\
\cline { 2 - 5 } & Prueba & 36.80 & 14.83 & 35.00 \\
\hline
\end{tabular}

Los resultados de este trabajo permitieron iniciar las obras necesarias para la ampliación del alcance del campo de pruebas de cortocircuito en transformadores de la Universidad del Valle.

\section{CONCLUSIONES}

Los resultados muestran que la compensación paralelo, es una alternativa viable para reducir los requerimientos de capacidad de corriente de un campo de pruebas de cortocircuito.

La comparación entre los resultados obtenidos en la simulación y la prueba, muestra una variación promedio de $1 \%$.

Con la compensación paralelo se obtuvo una disminución superior al $50 \%$ en la corriente suministrada por la fuente, sin afectar la corriente en el transformador.

A partir de estos resultados, se desarrolla el diseño de un sistema de compensación para emplearse con los diferentes transformadores a probar. Con esto se podrá certificar la calidad de un mayor número de transformadores fabricados en Colombia y países vecinos para soportar los esfuerzos electrodinámicos que se presentan bajo una condición de cortocircuito franco. El sector eléctrico se verá beneficiado por el aumento de la confiabilidad de los transformadores de distribución y la mejor calidad del servicio de energía eléctrica brindado a los usuarios.

\section{AGRADECIMIENTOS}

Los autores agradecen al Departamento Administrativo de Ciencia, Tecnología e Innovación en Colombia COLCIENCIAS, por su apoyo en el desarrollo de este trabajo a través del proyecto "Sistema resonante para realizar la prueba de cortocircuito franco en transformadores de distribución".

\section{REFERENCIAS}

Aponte G., Cadavid H., Castro F., Lozano C., y Lurie A. I., Colombian Experiences in Distribution Transformers Short Circuit Withstand Aptitud Test. 10th International Symposium on High Voltage Engineering, Montreal-Canadá, 25 al 29 de Agosto (1997).

Aponte G., Herrera W., González C. y Pleite J., Implementación de un Modelo de un Transformador Eléctrico para el Análisis de su Respuesta en Frecuencia, Revista Información Tecnológica, Vol. 22, No 4, 59-72 (2011). 
Fogelberg T., Short-Circuit Withstand Capability of Power Transformers, ABB ReviewTransformers and Substations, ISSN: 1013-3119, Vol 1, 24-28 (2008).

Fogelberg T., Leufkens P., Smeets R. y Paske L., Thirteen Years Test Experience with ShortCircuit Withstand Capability of Large Power Transformers, Articulo P501, Cigré 6th Southern Africa Regional Conference, 1-7, Ciudad del Cabo-Suráfrica, 17 al 21 de Agosto (2009).

Fortin M., Gerth J., McLaughlin R. y Riffon P, Short-circuit Strength and Short-circuit Testing of Power and Distribution Transformers, Technical Presentation-IEEE/PES Transformers Committee, Carolina del Norte-Estados Unidos, 17 de Marzo (2008).

Geno P., Calculations for Short Circuit Withstand Capability of a Distribution Transformer, International Journal of Engineering, Vol 9, No 3, 243-246 (2011).

Huang Z. y Xu W., A Practical Harmonic Resonance Guideline for Shunt Capacitor Applications, IEEE Transactions on Power Delivery, Vol. 18, No. 4, 1382-1387 (2003).

IEC 60076-3, International Electro technical Commission, Insulation Levels, Dielectric Tests and External Clearances in Air, 13-73, Ginebra-Suiza (2000).

IEC 60076-5, International Electrotechnical Commission, Power Transformers Ability to Withstand Short Circuit, 9-39, Ginebra-Suiza (2006).

IEEE C57.12.90, Institute of Electrical and Electronics Engineers, Standard Test Code for LiquidImmersed Distribution, Power, and Regulating Transformers, 56-61, Nueva York-Estados Unidos (2010).

Jardinetti R., O Sistema Elétrico Diretamente Enterrado (SEDE) Implantação, Monitoração e Automação o Caso da Cidade Universitária Armando de Salles Oliveira, Dissertação do Mestre em Energia, Universidade de São Paulo, Programa de Pós-Graduação em Energia (2002).

Khaparde S. y Kulkarni S., Transformer Engineering Design and Practice, $2^{\mathrm{a}}$ edición, 23-25. Marcel Dekker Inc, Nueva York-Estados Unidos (2004).

Maitra A. y Santoso S., Empirical Estimation of System Parallel Resonant Frequencies Using Capacitor Switching Transient Data, IEEE Transactions on Power Delivery, Vol. 20, No. 2, 11511153 (2005).

Martínez J. y de León F., Circuito Equivalente de un Transformador con Regulación, Ingeniare Revista Chilena de Ingeniería, Vol 19, No 1, 93-109 (2011).

Natarajan R., Power System Capacitors, $2^{a}$ edición, 402-405. Taylor \& Francis Group, Carolina, Estados Unidos (2005).

NTC 532, Instituto Colombiano de Normas Técnicas y Certificación, Transformadores - Aptitud para Soportar el Cortocircuito, 1-20, Bogotá, Colombia, (1999).

NTC 837, Instituto Colombiano de Normas Técnicas y Certificación, Transformadores - Ensayo del Dieléctrico, 1-12, Bogotá, Colombia (1997). 